\title{
DEVELOPMENT OF A HYBRID FUZZY GENETIC ALGORITHM MODEL FOR SOLVING TRANSPORTATION SCHEDULING PROBLEM
}

\author{
H.C.W. Lau \\ Dilupa Nakandala \\ Li Zhao \\ University of Western Sydney, Penrith, New South Wales, Australia
}

\begin{abstract}
There has been an increasing public demand for passenger rail service in the recent times leading to a strong focus on the need for effective and efficient use of resources and managing the increasing passenger requirements, service reliability and variability by the railway management. Whilst shortening the passengers' waiting and travelling time is important for commuter satisfaction, lowering operational costs is equally important for railway management. Hence, effective and cost optimised train scheduling based on the dynamic passenger demand is one of the main issues for passenger railway management. Although the passenger railway scheduling problem has received attention in operations research in recent years, there is limited literature investigating the adoption of practical approaches that capitalize on the merits of mathematical modeling and search algorithms for effective cost optimization. This paper develops a hybrid fuzzy logic based genetic algorithm model to solve the multi-objective passenger railway scheduling problem aiming to optimize total operational costs at a satisfactory level of customer service. This hybrid approach integrates genetic algorithm with the fuzzy logic approach which uses the fuzzy controller to determine the crossover rate and mutation rate in genetic algorithm approach in the optimization process. The numerical study demonstrates the improvement of the proposed hybrid approach, and the fuzzy genetic algorithm has demonstrated its effectiveness to generate better results than standard genetic algorithm and other traditional heuristic approaches, such as simulated annealing.
\end{abstract}

Keyword: passenger railway scheduling; fuzzy logic approach; genetic algorithm; customer service optimization

Manuscript first received/Recebido em: 20/05/2014 Manuscript accepted/Aprovado em: 19/10/2015

Address for correspondence / Endereço para correspondência

H.C.W. Lau, Corresponding author. School of Business, University of Western Sydney, Locked Bag 1797, Penrith, New South Wales 2751, Australia, Tel.: +61 2 96859488, Mobile: +61 459123038. Email:H.Lau@uws.edu.au.

Dilupa Nakandala, School of Business, University of Western Sydney, Locked Bag 1797, Penrith, New South Wales 2751, Australia, Tel.: +61 2 96859488, E-mail:D.Nakandala@westernsydney.edu.au.

Li Zhao, School of Business, University of Western Sydney, Locked Bag 1797, Penrith, New South Wales 2751, Australia, Tel.: +61 296859488

Published by/ Publicado por: TECSI FEA USP - 2015 All rights reserved. 


\section{INTRODUCTION}

Passenger railway companies are facing increasing demands nowadays, such as the need for effective and efficient use of resources, the competitive transportation markets, the increasing passenger requirements, the need for service reliability and availability (Fay, 2000). Competing requirements of the efficient use of resources and meeting passenger demands make passenger train scheduling a difficult task. The passenger train schedule planning is important to railway operations due to the fact that the management of passenger train service is mainly based on regular train schedules (Chang, et al., 2000). One of the main issues of passenger train scheduling is the dynamic nature of passenger flows which are uneven in different time-periods of the day (Huang and Niu,2012). How to make suitable train schedule based on the dynamic demand is one of the main problems for railway management. Whilst shortening the passengers' waiting and travelling time is necessary to improve passenger satisfaction, lowering operational costs is also necessary for railway management. The general problem that passenger railway companies face nowadays is how to maintain a balance between customer service and operational costs, higher operational costs are usually considered to have better customer service level (Chang, et al., 2000). However, no profit making corporation would maximize the total operational costs for perfect service. They would prefer to find an optimum solution which provides an acceptable standard of customer service within limited resources.

Passenger railway scheduling problem has received considerable attention from both practitioners and scholars. However, most of the work is concerned with the use of mathematical programming such as linear programming, which fails to incorporate reallife characteristics and is not easy to apply in real situations. There is limited literature regarding the adoption of a practical approach which capitalizes on the merits of mathematical modeling and heuristics search algorithms to obtain optimized outcomes in an effective way. This paper contributes to the literature by using novel heuristic optimization approaches in the multi-objective passenger railway scheduling problem. Well-developed optimization techniques, standard genetic algorithm (GA) method and hybrid fuzzy GA method, which focus on finding the nearly optimal solutions based on a stochastic search technique are adopted to optimize the total operational costs while maintaining the customer service level based on a list of known constraints. In this research, we proposed a pragmatic approach, taking advantage of readily available and easy to use solutions to put our suggested approach in practice, with specific skills and training. The guidelines and steps we have provided in this paper can be adopted by practitioners to incorporate in solving their scheduling problem with going through complex computation.

The rest of the paper is organized as follows. Section 2 reviews the relevant literature. Section 3 presents the formulation of the model. The standard GA method and fuzzy GA method are proposed in section 3. The application of the fuzzy GA model is illustrated with a numerical study in Section 4, followed by the discussion of the results in section 5 . The last section highlights the findings and suggestions for future research.

\section{LITERATURE REVIEW}

Passenger railway transportation has received considerable attention in operations research in recent years (Huisman, et al., 2005). Most of the earlier studies in 
train planning and scheduling used a single-objective approach, focusing on either enhancing service level or minimizing operational costs (Chang et al., 2000). Later on, many scholars found the importance of matching customer demand and cost reduction requirements. Multi-objective passenger railway optimization has been under study with results published in various journals. For example, Chang et al. (2000)'s research aims to minimize the operator's total operational costs and minimizing the passenger's total travel time loss. Huisman et al. (2005) argued that not only the timetable itself is important, the reliability of the timetable is even more important. Service to the passengers, efficiency and robustness are three main objectives for passenger railway transportations. Niu (2011) formulated a nonlinear programming model to minimize the overall waiting time and in-vehicle crowding costs. Huang and Niu (2012) formulated an optimized model to maximize passenger satisfaction by shortening the total passenger-time and maximum railway company satisfaction through the lowering of fuel consumption costs.

As railway optimization problem is complicated and it is usually considered as a mix integer linear programming problem, how to find solutions for this problem is widely discussed in the past research. Caprara (2015) classified the railway planning problems to timetabling and assignment problems. He modeled them as mix integer linear programming, and discussed the solution methods and modelling issues. Most of the previous work is concerned with the use of linear programming and other optimization approaches using heuristics and computational intelligence methods. For example, He et al. (2000) developed a fuzzy dispatching model and genetic algorithm to assist the coordination among multi-objective decisions in rail yards dispatching. Vromons and Kroon (2004) described a stochastic timetable optimization model, providing a linear programming model with minimal average delay under certain disruptions. Huisman et al. (2005) gave an overview of operations research models and techniques used in passenger railway transportation, dividing the planning problems into strategic, tactical and operational phases. They pointed out that heuristic approaches are required for short-term railway scheduling problem and real-time control of the passenger railways. Niu (2011) formulated a nonlinear programming model for the skip-station scheduling problem for a congested transit line. Schindl and Zufferey (2015) considered a refueling problem in a railway network and decomposed it in two optimization levels. They proposed a learning tabu search method to solve this problem and the results show good performance of learning tabu search.

Despite the efforts past research has made, all these techniques are not easy to apply in real situations, and they also require expertise and special skills for implementation. There is limited literature investigating the adoption of practical approaches that capitalize on the merits of mathematical modeling and search algorithms for effective transportation scheduling optimizations. Minimum costs against certain number of runs of train schedule can be found mathematically. However, in reallife scenarios, there is a list of constraints such as the limited number of trains, the available capacity of the station, the complexity of the various costs and the uncertainty of passenger flow. More work still needs to be done in using more practical approaches which capitalize on the merits of mathematical modeling and heuristic search approaches to solve the passenger train planning and scheduling problem. 


\section{PROPOSED APPROACH}

This paper develops a hybrid method for the passenger railway scheduling that minimizes the total operational costs and maintains customer service level for railway companies. Given that the passenger arrival changes during the day and the number of passengers and trains is large, the manual processing methods become impractical for determining the best schedule of the passenger trains.

The following notation is used in the model development.

$\mathrm{TC}=$ Total operational costs in the objective function

SC =Service cost assigned according to the respective waiting time at stations

$\mathrm{VC}=$ Variable cost of operation

FC $=$ Fixed cost of operation

$N C=$ The average number of waiting passengers in station

$N \mathrm{C}_{\mathrm{i}} \quad=$ The number of waiting passengers in the $\mathrm{i}^{\text {th }}$ minute.

$\mathrm{d}_{\mathrm{i}} \quad=$ Number of traveling passengers in the $\mathrm{i}^{\text {th }}$ minute

$n_{i} \quad=$ The schedule of train running in the $\mathrm{i}^{\text {th }}$ minute.

- $n_{i}=1$ means there is a train scheduled in the $\mathrm{i}^{\text {th }}$ minute;

- $n_{i}=0$ means there is no train scheduled in the $\mathrm{i}^{\text {th }}$ minute.

$W_{1} \quad$ =Weighting assigned for service costs of fitness function.

$W_{2} \quad$ =Weighting assigned for variable costs of fitness function.

$W_{3} \quad=$ Weighting assigned for fixed costs of fitness function.

$p_{c} \quad=$ crossover rate

$p_{m} \quad=$ mutation rate.

$N_{g} \quad$ =maximum number of generations.

$M_{T} \quad=$ the maximum frequency of train schedules of a given period.

$M_{C} \quad$ =the capacity of the respective station.

Total passenger railway operational costs (TC) includes elements such as variable cost (VC), service cost (SC), and fixed cost (FC),

$$
\mathrm{TC}=\mathrm{VC}+\mathrm{FC}+\mathrm{SC}(1)
$$

The variable cost (VC) varies at each run of train schedule and includes costs related to human resources, power resources, and maintenance etc.

The fixed cost (FC) is the cost associated with the basic requirements of operating resources of its transportation system such as administration expenses. As it is not associated with the number of run of trains, it is a constant in the cost function. 
The service cost (SC) is different from the fixed and variable cost and denotes the cost incurred due to the passengers' waiting time at stations. The longer the waiting time, the higher the service cost. Cost per minute or certain time period is estimated and assigned to each passenger for calculating the final service cost.

The decision variable of our objective function is the schedule of the run of train in each minute $n_{i}$ during the $\mathrm{T}$ period. The weighting $\left(\mathrm{W}_{\mathrm{i}}\right)$ is assigned to each $\mathrm{i}^{\text {th }}$ element of the objective function for different emphasis. This allows controlling the emphasis on each cost element; customer satisfaction could be given more priority by giving $\mathrm{W}_{1}$ more weight. Thus, the total operational costs are shown as follows:

$$
T C=W_{1}(N C * S C)+W_{2}\left(\sum_{i=1}^{T} n_{i} * V C\right)+W_{3} * F C
$$

Constraints of the objective function include the following four aspects:

First, the number of runs of train schedules cannot exceed the limit of the maximum frequency of train schedules of a given period. Thus:

$$
\sum_{i=1}^{T} n_{i} \leq M_{T}(3)
$$

Second, the number of waiting passengers is limited by the available capacity of respective station. Thus:

$$
N C \leq M_{C}
$$

Third, the weighting of all components must be equal to 1 . Thus:

$$
W_{1}+W_{2}+W_{3}=1
$$
trains

Fourth, the decision variables should be positive integers for runs of scheduled

$$
n_{i}=0,1
$$

\subsection{GA method}

GA method is a search method used to find approximate solutions for optimization problems which use techniques inspired by evolutionary biology such as selection, crossover and mutation (Peng and Song, 2010). The standard process of GA is an iterative process loosely based on natural selection, crossover and mutation (shown in Fig.1). The objective function values are evaluated to compute the level of fitness of each possible solution. Some individual solutions are randomly selected for reproduction where the probability of selection is proportional to their level of fitness values. By using the crossover and mutation operators, new offspring are generated and the objective function values are evaluated for each generation in order to determine the new fitness values and consequent appropriate replacements of the solutions with the minimum fitness values by the generated offspring solutions to create a new generation. This process is continued until the pre-set generation threshold is met and at the end point the best chromosome is selected as the preferred solution for implementation. 


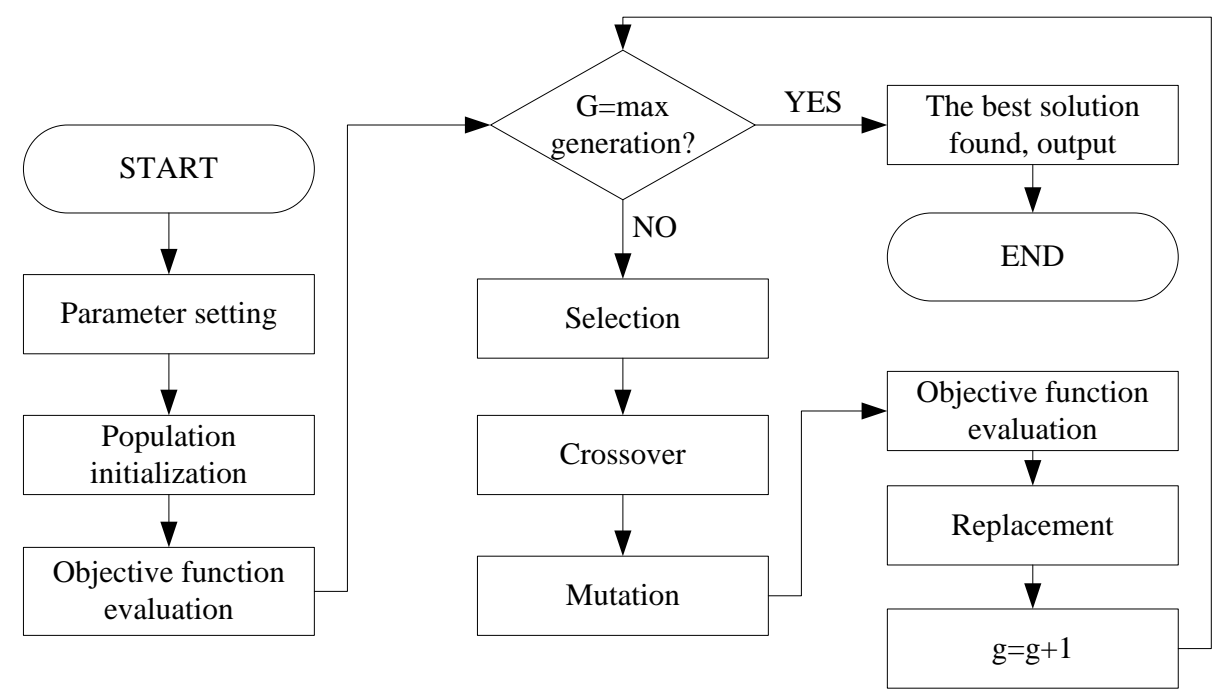

Fig.1. The standard workflow of GA

Crossover is adopted to exchange information between two parents' chromosomes for genetic exploration. Not all chromosomes are chosen for crossover. Whether a chromosome is selected or not determined by the crossover rate $p_{c}$, with a preset value. In the crossover operation, the mating pairs of chromosomes produce two offspring chromosomes. If no crossover happens, the offspring chromosomes are the same as their parents' chromosomes (Taleizadeh, et al., 2013). In the reproduction process, either one-point crossover or two-point crossover is used (Della Croce et al 1995) and this paper adopts the one-point crossover method.

Mutation is the second genetic operator which provides diversity into future generations so as to prevent them from falling into local optimal. Whether a chromosome is selected, it is determined by the mutation rate $p_{m}$. A common method is to randomly assign mutation probability values for each gene, and then compare the probability value with the preset mutation rate to determine whether a particular gene will be mutated.

When offspring replace their parent chromosomes, fitness values are calculated for chromosomes in the mating pool. The two least objective function values are replaced with the two best of the new mating pool objective values. The difference between the original solution and the new set provides the degree of improvement of the solution. This process is repeated until the generation counter reaches the pre-set maximum generation value of $\mathrm{Ng}$ and the passenger railway schedule is done.

Fig. 2 represents the chromosome structure of our study. Each available scheduled train is assigned with binary gene. " 1 " represents present while " 0 " represents absence of respective train.

\begin{tabular}{|c|c|c|c|c|c|c|c|}
$\mathrm{n}_{1}$ & $\mathrm{n}_{2}$ & $\mathrm{n}_{3}$ & \multicolumn{1}{c}{$\mathrm{n}_{4}$} & \multicolumn{2}{c}{$\mathrm{n}_{\mathrm{T}-1}$} & $\mathrm{n}_{\mathrm{T}}$ \\
\hline 1 & 0 & 0 & 0 & $\cdots$ & 0 & 1 \\
\hline
\end{tabular}

Fig.2. chromosome structure of the study 


\subsection{Fuzzy GA method}

There are possible performance limitations of the standard GA method, such as the overall effectiveness pre-set parameter value that limits the flexibility of the method. If the pre-set parameters, such as crossover rate $\left(p_{c}\right)$ and mutation rate $\left(p_{m}\right)$ are inappropriate, the fittest solution cannot be found. In standard GA, $p_{c}$ and $p_{m}$ are fixed, which may influence the performance of the algorithm (Peng and Song, 2010). Many scholars suggest improving the standard GA method by changing the pre-set parameters, such as $p_{c}$ and $p_{m}$ (Maiti, 2011). However, as Peng and Song (2010) pointed out, how to change the value of $p_{c}$ and $p_{m}$ in the process is quite complex, as they cannot be defined as precise formula. Fuzzy theory is used in literature to integrate with standard GA to improve the performance of GA (Peng and Song, 2010; Maiti, 2011; Taleizadeh, et al., 2013).

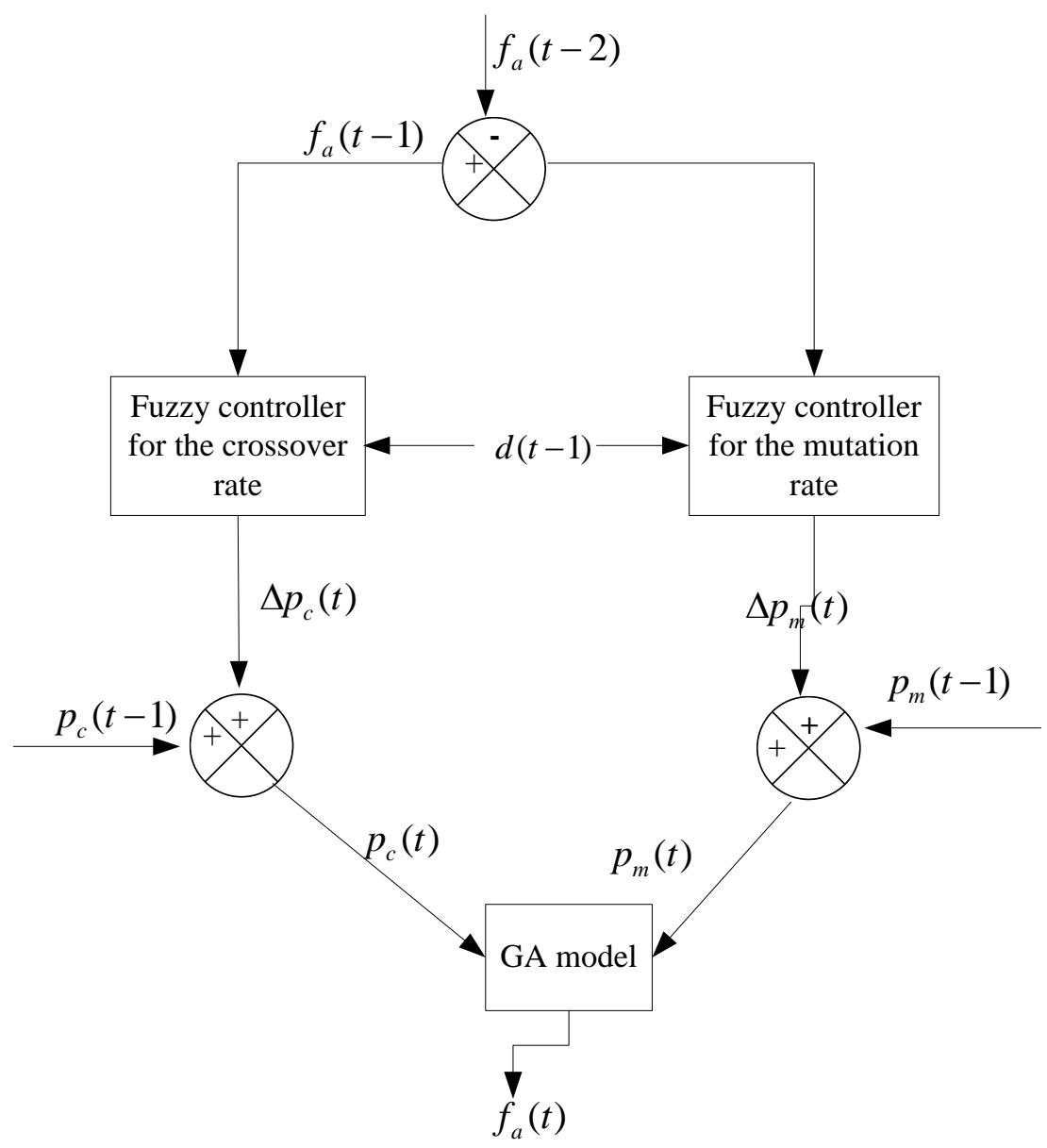

Fig.3. The fuzzy GA method

Adapted from Nakandala et al. (2014)

Adapted from Nakandala et al. (2014)'s research, the process of fuzzy GA approach is shown in Fig.3. Changes are performed on the two pre-set parameters: crossover rate $p_{c}$ and mutation rate $p_{m}$. The fuzzy controllers are used to determine the size of required changes. The input variables for the fuzzy controllers include the degree 
of fitness difference of chromosome generation $\Delta f$ and the degree of population diversity at generation ( $\mathrm{t}-1)$, that is $d(t-1)$.

Let $f_{a}(t-1)$ and $f_{a}(t-2)$ be the average fitness values at generation $(\mathrm{t}-1)$ and $(\mathrm{t}-2)$. $\Delta f$ is calculated using the following formula (7).

$$
\Delta f=\frac{f_{a}(t-1)-f_{a}(t-2)}{f_{a}(t-2)}
$$

It is then categorized relatively, and the corresponding fuzzy sets are characterized by its element of $\Delta f_{i}$ as below and the membership function $\mu_{\Delta f}\left(\Delta f_{i}\right)$. The fuzzy set of $\mu_{\Delta f}\left(\Delta f_{i}\right)$ is shown in Fig.4.

$$
\Delta f_{i}=\{N L R, N L, N M, N S, Z, P S, P M, P L, P L R\}
$$

Where NLR means Negative Larger; NLmeans Negative Large; NMmeans Negative Medium; NSmeans Negative Small; Zis Zero; PSis Positive Small; PMis Positive medium; PL is Positive Large; and PLR is Positive Larger.

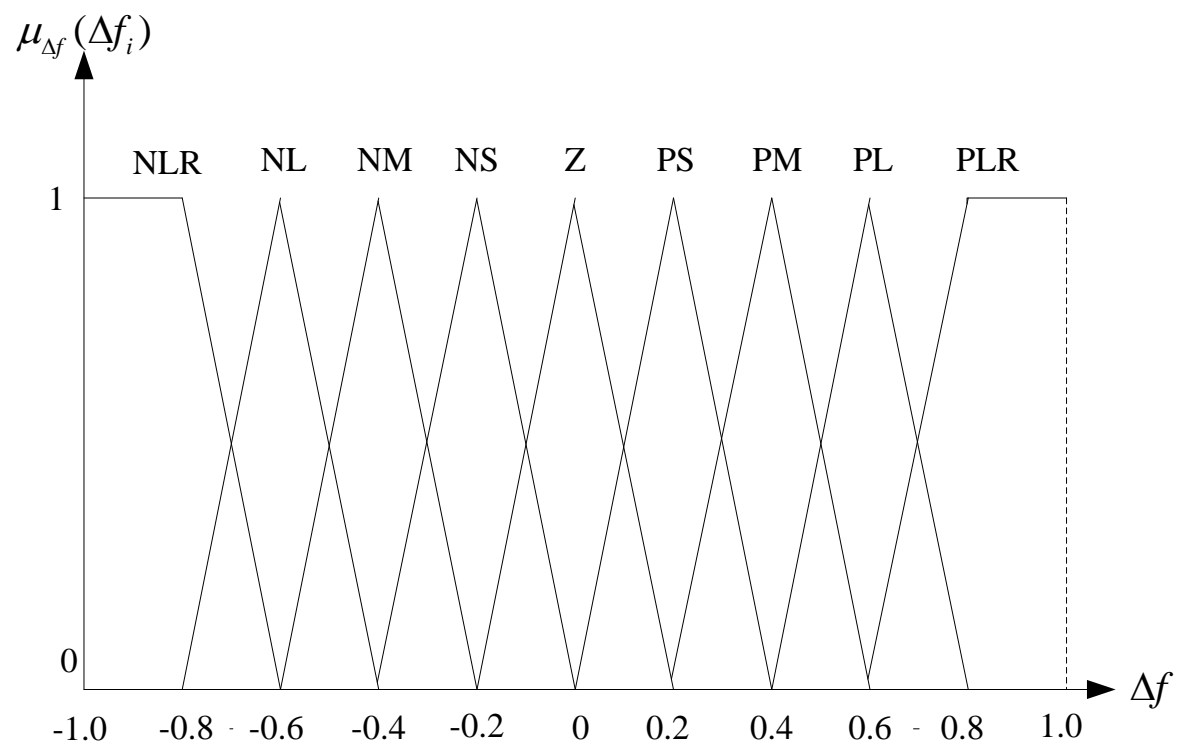

Fig.4. The fuzzy set of $\mu_{\Delta f}\left(\Delta f_{i}\right)$

The physical meaning of $d$ is to evaluate the average of bit difference of all pairs of chromosomes in the population and it can be calculated by using the following formula (8):

$$
d=\frac{1}{\left[\frac{N(N-1)}{2}\right]} \sum_{i=1}^{N} \sum_{j=i+1}^{N} \sum_{k=1}^{M} \frac{\delta\left(g_{i k}, g_{j k}\right)}{M}
$$

Where $\mathrm{N}$ is the total number of chromosomes in the population, $\mathrm{M}$ is the chromosome length, $g_{i k}$ is the value of $\mathrm{k}^{\text {th }}$ gene of $\mathrm{i}^{\text {th }}$ chromosome, and $\delta\left(g_{i k}, g_{j k}\right)=1$ if $g_{i k} \neq g_{j k}$ 
$d(t-1)$ is categorized by its elements of $d_{i}$ and the membership function of $\mu_{d}\left(d_{i}\right)$ (shown in Fig.5). Its elements of $d_{i}$ are as below:

$d_{i}=\{V S, S, S S, L M, M, U M, S L, L, V L\}$

Where VSis Very Small; Sis Small; SSis Slightly Small, LMis Lower Medium; Mis Medium; UM is Upper Medium; SL is Slightly Large; L is Large; and VLis Very Large.

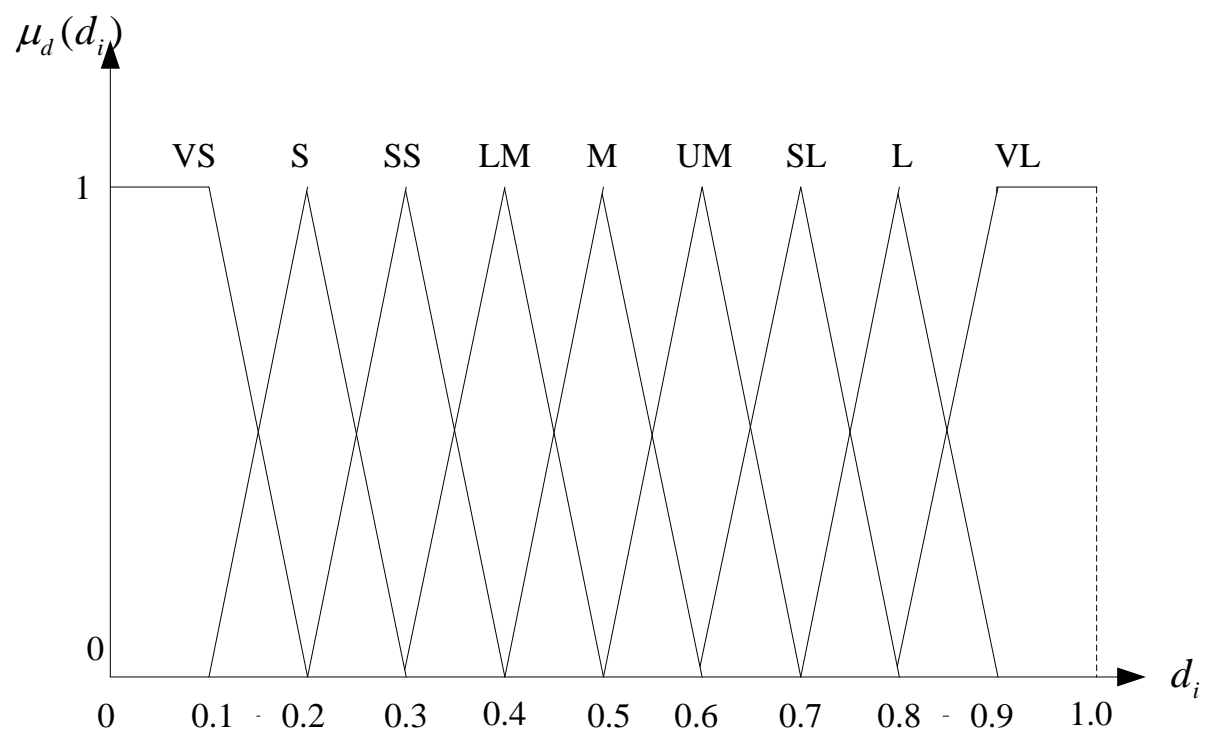

Fig.5. The fuzzy set of $\mu_{d}\left(d_{i}\right)$

The two fuzzy controllers determine the change of the crossover rate and mutation rate, that is the values of $\Delta p_{c}(t)$ and $\Delta p_{m}(t) . \Delta p_{c}(t)$ and $\Delta p_{m}(t)$ are presented as fuzzy sets in Fig.6 and the Fig. 7.

$$
\Delta p_{c}(t)=\{N L R, N L, N M, N S, Z, P S, P M, P L, P L R\}
$$

and $\Delta p_{m}(t)=\{N L R, N L, N M, N S, Z, P S, P M, P L, P L R\}$

Where NLR= Negative Larger; NL = Negative Large; NM = Negative Medium; NS = Negative Small; Z = Zero; PS = Positive Small; PM = Positive medium; PL = Positive Large; and PLR = Positive Larger. 


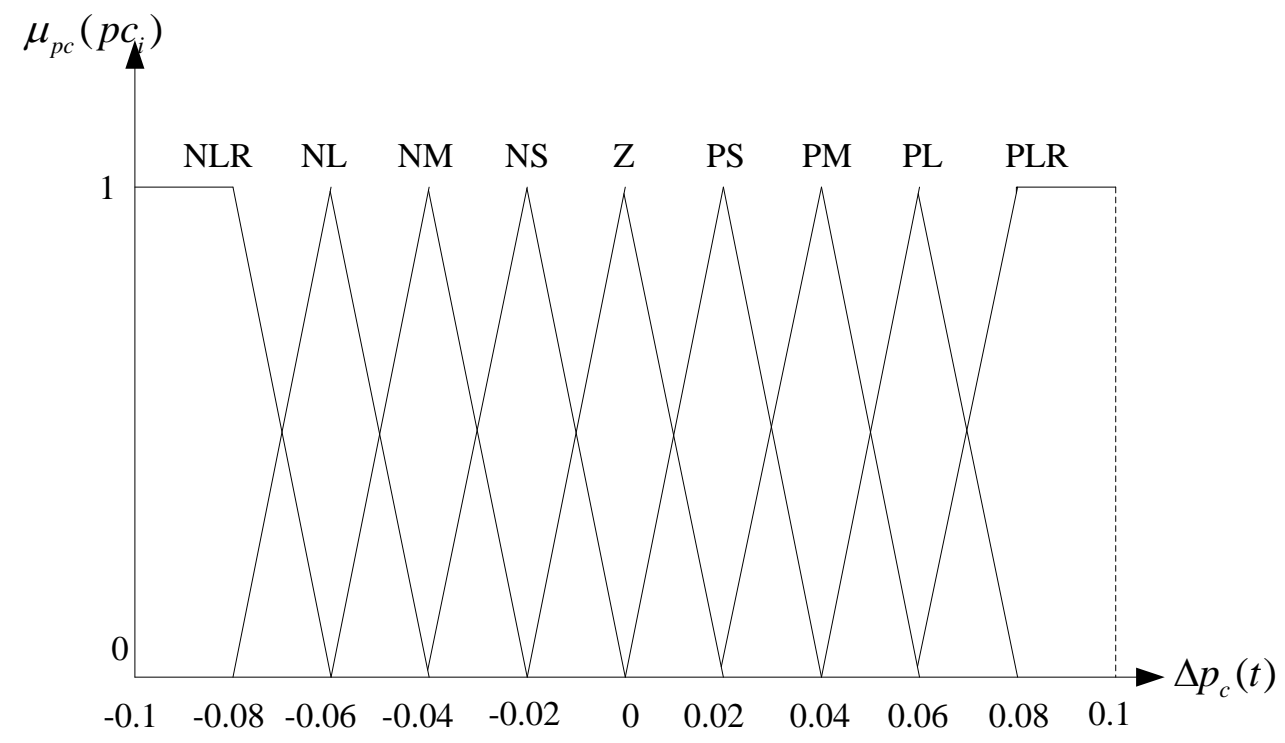

Fig.6. The fuzzy set of $\mu_{p c}\left(p c_{i}\right)$

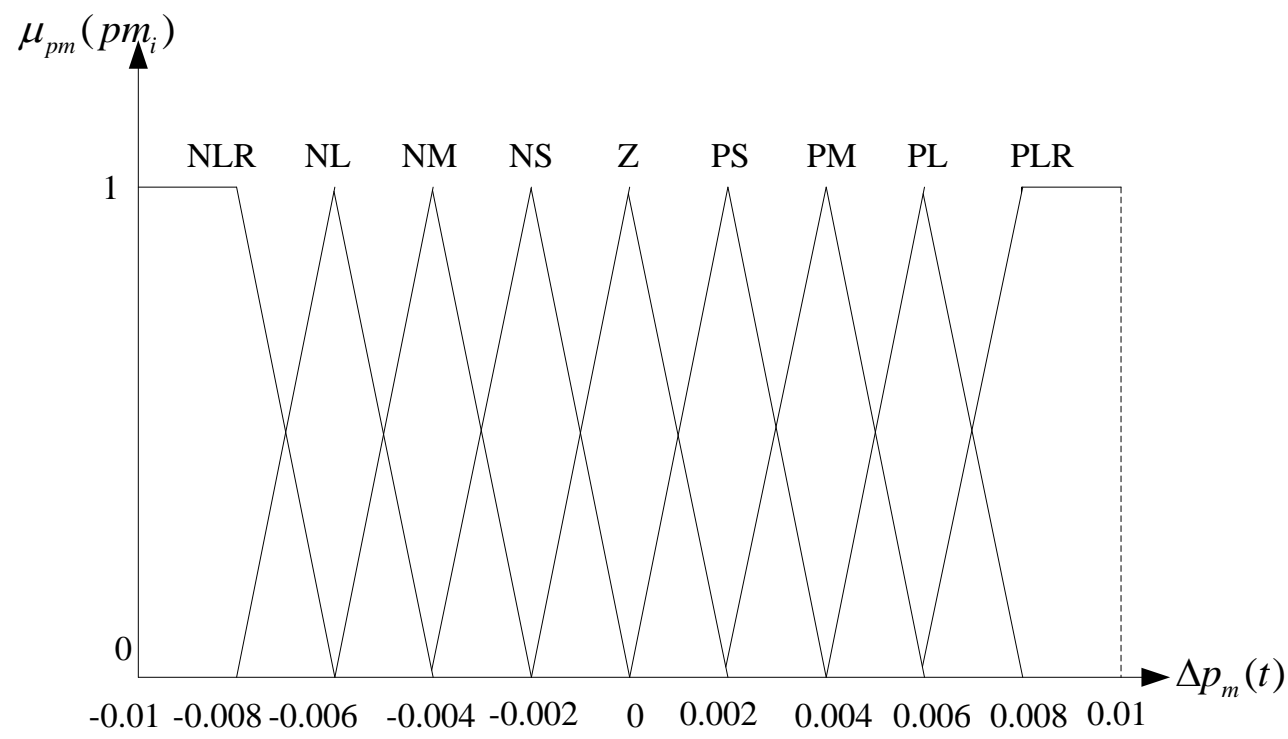

Fig.7. The fuzzy set of $\mu_{p m}\left(p m_{i}\right)$

The main advantage of this hybrid fuzzy GA approach is that it incorporates the experts' tacit knowledge into the change of the preset parameters $\Delta p_{c}(t)$ and $\Delta p_{m}(t)$ as the change of these two parameters is based on tacit knowledge of the field experts who have experience from similar situations. The process involves studying and understanding the situation in order to design the relevant appropriate fuzzy system, identifying the appropriate expertise and input data types (Lau and Dwight, 2012). 
Table 1. The fuzzy rule table for crossover rate change

\begin{tabular}{llllllllll}
\hline \multicolumn{1}{c}{$\Delta \mathrm{d}_{\mathrm{n}(\mathrm{t}-1)}$} & NLR & NL & NM & NS & Z & PS & PM & PL & PLR \\
\hline VL & PLR & PLR & PL & PL & PM & PM & PS & PS & Z \\
L & PLR & PL & PL & PM & PM & PS & PS & Z & NS \\
SL & PL & PL & PM & PM & PS & PS & Z & NS & NS \\
UM & PL & PM & PM & PS & PS & Z & NS & NS & NM \\
M & PM & PM & PS & PS & Z & NS & NS & NM & NM \\
LM & PM & PS & PS & Z & NS & NS & NM & NM & NL \\
SS & PS & PS & Z & NS & NS & NM & NM & NL & NL \\
S & PS & Z & NS & NM & NM & NM & NL & NL & NLR \\
VS & Z & NS & NS & NM & NM & NL & NL & NLR & NLR \\
\hline
\end{tabular}

In the fuzzy inference engine, the IF-THEN fuzzy rules are developed based on the expert knowledge and historical data. Table 1 and Table 2 show the rule sets for generating the output of $\Delta p_{c}(t)$ and $\Delta p_{m}(t)$. According to the IF-part of the rules, composition result of each rule is calculated, and the results are then put into the implication process to determine the Then-part output fuzzy set with different implication operators. For example, if the degree of fitness difference of chromosome generation $\Delta f$ is NLR and the degree of population diversity at generation (t-1) is VL, the crossover rate change would be PLR.

Table 2. The fuzzy rule table for mutation rate change

\begin{tabular}{llllllllll}
\hline \multicolumn{1}{c}{$\Delta(\mathrm{t}-1)$} & NLR & NL & NM & NS & Z & PS & PM & PL & PLR \\
\hline VL & NLR & NLR & NL & NL & NM & NM & NS & NS & Z \\
L & NLR & NL & NL & NM & NM & NS & NS & Z & PS \\
SL & NL & NL & NM & NM & NS & NS & Z & PS & PS \\
UM & NL & NM & NM & NS & NS & Z & PS & PS & PM \\
M & NM & NM & NS & NS & Z & PS & PS & PM & PM \\
LM & NM & NS & NS & Z & PS & PS & PM & PM & PL \\
SS & NS & NS & Z & PS & PS & PM & PM & PL & PL \\
S & NS & Z & PS & PS & PM & PM & PL & PL & PLR \\
VS & Z & PS & PS & PM & PM & PL & PL & PLR & PLR \\
\hline
\end{tabular}

In the last stage of the fuzzy logic approach, defuzzification is performed to convert the fuzzy values into crisp values or linguistic values, which is the inverse 
process of fuzzification. The crisp values of $\Delta p_{c}(t)$ and $\Delta p_{m}(t)$ are got from the defuzzification stage and used for the adjustment of the crossover rate and mutation rate at generation $\mathrm{t}$.

At generation $\mathrm{t}$, the crossover rate and mutation rate will be adjusted as shown in formula (9) and (10), and GA model will use $p_{c}(t)$ and $p_{m}(t)$ instead of $p_{c}(t-1)$ and $p_{m}(t-1)$ to proceed the search at generation $\mathrm{t}$.

$$
\begin{array}{r}
p_{c}(t)=p_{c}(t-1)+\Delta p_{c}(t) \\
p_{m}(t)=p_{m}(t-1)+\Delta p_{m}(t)
\end{array}
$$

\section{NUMERICAL STUDY}

We select one of the busiest stations, named station A, in the railway line, which has a large number of passengers during the morning peak hours. It is assumed that trains are available for one minute time interval, thus at most 20 trains can be run for the given time period 20 minutes to meet demand. Another assumption is that the maximum capacity of the train is 500 passengers.

Table3. First 20 train arrival rate with one minute interval and possible train schedules

\begin{tabular}{cccc}
\hline Time & $\begin{array}{c}\text { Demand } \\
\left(d_{i}\right)\end{array}$ & $\begin{array}{c}\text { Schedule } \\
\left(n_{i}\right)\end{array}$ & $\begin{array}{c}\text { Waiting passenger } \\
\left(N C_{i}>=0\right)\end{array}$ \\
\hline 8:00:00 & 317 & $n_{1}$ & $317-500^{*} n_{1}$ \\
8:01:00 & 230 & $n_{2}$ & $N C_{1}+230-500^{*} n_{2}$ \\
$8: 02: 00$ & 222 & $n_{3}$ & $N C_{2}+222-500^{*} n_{3}$ \\
$8: 03: 00$ & 178 & $n_{4}$ & $N C_{3}+178-500^{*} n_{4}$ \\
$8: 04: 00$ & 362 & $n_{5}$ & $N C_{4}+362-500^{*} n_{5}$ \\
$8: 05: 00$ & 253 & $n_{6}$ & $N C_{5}+253-500^{*} n_{6}$ \\
$8: 06: 00$ & 287 & $n_{7}$ & $N C_{6}+287-500^{*} n_{7}$ \\
$8: 07: 00$ & 316 & $n_{8}$ & $N C_{7}+316-500^{*} n_{8}$ \\
$8: 08: 00$ & 252 & $n_{9}$ & $N C_{8}+252-500^{*} n_{9}$ \\
$8: 09: 00$ & 217 & $n_{10}$ & $N C_{9}+217-500^{*} n_{10}$ \\
$8: 10: 00$ & 296 & $n_{11}$ & $N C_{10}+296-500^{*} n_{11}$ \\
$8: 11: 00$ & 301 & $n_{12}$ & $N C_{11}+301-500^{*} n_{12}$ \\
$8: 12: 00$ & 340 & $n_{13}$ & $N C_{12}+340-500^{*} n_{13}$ \\
$8: 13: 00$ & 206 & $n_{14}$ & $N C_{13}+206-500^{*} n_{14}$ \\
\hline
\end{tabular}




\begin{tabular}{llll}
\hline $8: 14: 00$ & 234 & $n_{15}$ & $N C_{14}+234-500^{*} n_{15}$ \\
$8: 15: 00$ & 265 & $n_{16}$ & $N C_{15}+265-500^{*} n_{16}$ \\
$8: 16: 00$ & 249 & $n_{17}$ & $N C_{16}+249-500^{*} n_{17}$ \\
$8: 17: 00$ & 215 & $n_{18}$ & $N C_{17}+215-500 * n_{18}$ \\
$8: 18: 00$ & 230 & $n_{19}$ & $N C_{18}+230-500 * n_{19}$ \\
$8: 19: 00$ & 267 & $n_{20}$ & $N C_{19}+267-500 * n_{20}$ \\
\hline
\end{tabular}

The number of passengers waiting equals to the number of passengers who cannot take the train as it is fully occupied or there is no train when they arrive in the station and they need to wait for the next train. By a simple calculation, number of waiting passengers in $\mathrm{i}^{\text {th }}$ minute $\left(N C_{i}\right)=$ number of waiting passengers in $(\mathrm{i}-1)^{\text {th }}$ minute $\left(N C_{i-1}\right)+$ number of traveling passengers in $\mathrm{i}^{\text {th }}$ minute $\left(d_{i}\right)$ - travelled passenger in $\mathrm{i}^{\text {th }}$ minute. Thus:

$$
N C_{i}=\left\{\begin{array}{r}
N C_{i-1}+d_{i}-500 * n_{i} \text { if } N C_{i}>0 \\
0 \text { otherwise }
\end{array}\right.
$$

The service cost, variable cost and fixed cost coefficient are shown in the following table. Moreover, we assume $\mathrm{w} 1=\mathrm{w} 2=\mathrm{w} 3=1 / 3$.

Service cost (Incurred time) $\quad: 20$

Variable cost (power, staff, and maintenance, etc.) : 10,000

Fixed cost (administration) $\quad: 50,000$

The Fitness function calculation is shown in formula (12):

$$
\mathrm{TC}=1 / 3 *\left(20 * \sum_{i=1}^{20} N C_{i}+10000 * \sum_{i=1}^{20} n_{i}+50000\right)
$$

Subject to:

$$
\begin{array}{r}
\sum_{i=1}^{20} n_{i} \leq 20 \\
n_{i}=0,1(14) \\
N C_{i} \leq 1000
\end{array}
$$

\subsection{Simulation runs using the standard GA method}

Using standard GA method (The preset values of GA method are shown in Table 4), ten simulation runs were conducted and the results are shown in Table 5. Each simulation was run on different initial schedule of trains set randomly in the GA model. 
Table 4. Parameter pre-set values.

\begin{tabular}{ll}
\hline Parameter & Value \\
\hline Population size & 20 \\
Maximum generations & 500 \\
Crossover probability & 0.80 \\
Mutation probability & 0.005 \\
\hline
\end{tabular}

As shown in Table 5, the average fitness value of the 10 simulation runs is 72150.8 , with around 129 passengers waiting in each minute and 12 trains running in 20 minutes.

Table 5. Results for 10 simulation runs of standard GA.

\begin{tabular}{lccc}
\hline Runs & Fitness values & Average waiting passenger & Total number of trains \\
\hline 1 & 72447 & 118 & 12 \\
2 & 72200 & 142 & 11 \\
3 & 71873 & 139 & 11 \\
4 & 72260 & 117 & 12 \\
5 & 71687 & 138 & 11 \\
6 & 73560 & 127 & 12 \\
7 & 71627 & 112 & 12 \\
8 & 71740 & 113 & 12 \\
9 & 72227 & 142 & 11 \\
10 & 71887 & 139 & 11 \\
Average & 72150.8 & 128.7 & 11.5 \\
\hline
\end{tabular}

\subsection{Simulation runs using the hybrid fuzzy GA method}

Based on the standard GA method, the values of the input variables of degree of fitness difference $(\Delta f)$ and the degree of population diversity (d) are calculated and the values are 0.00068 and 0.051 .

$$
\begin{gathered}
\Delta f=\frac{f_{a}(t-1)-f_{a}(t-2)}{f_{a}(t-2)}=\frac{73740-73690}{73690}=0.00068 \\
d=\frac{1}{\left[\frac{N(N-1)}{2}\right]} \sum_{i=1}^{N} \sum_{j=i+1}^{N} \sum_{k=1}^{M} \frac{\delta\left(g_{i k} g_{j k}\right)}{M}=0.051
\end{gathered}
$$

The membership functions for each variable are:

$$
\mu_{z}=\left\{\begin{array}{rr}
(1 / 0.2)(f+0.2) & -0.2 \leq f \leq 0 \\
(-1 / 0.2)(f-0.2) & 0 \leq f \leq 0.2
\end{array}\right.
$$




$$
\begin{gathered}
\mu_{P S}=\left\{\begin{array}{rr}
(1 / 0.2)(f-0.0) & 0 \leq f \leq 0.2 \\
(-1 / 0.2)(f-0.4) & 0.2 \leq f \leq 0.4
\end{array}\right. \\
\mu_{V S}=\left\{\begin{array}{rr}
1 & 0 \leq d \leq 0.1 \\
(-1 / 0.1)(d-0.2) & 0.1 \leq d \leq 0.2
\end{array}\right.
\end{gathered}
$$

The crisp input values are fuzzified based on the membership functions for each input. The variable $\Delta f$ cuts the $\mathrm{Z}$ predicate at 0.9966 and PS predicate at 0.0034 . Similarly, the variable $d$ cuts the VS predicate at 1 . Based on the membership values of the two inputs, four rules are generated from the fuzzy rule blocks (shown in Table 6).

Table 6. Rule generation based on the membership function of $\Delta f$ and d.

\begin{tabular}{lll}
\hline Rule No & "IF" & "THEN" \\
\hline 1 & $\begin{array}{l}\text { The degree of fitness difference IS Zero AND } \\
\text { the degree of population diversity IS very small }\end{array}$ & $\begin{array}{l}\text { Crossover change rate } \\
\text { is Negative medium }\end{array}$ \\
& $\begin{array}{l}\text { The degree of fitness difference IS positive small } \\
\text { AND } \\
\text { the degree of population diversity IS very small }\end{array}$ & $\begin{array}{l}\text { Crossover change rate } \\
\text { is Negative large }\end{array}$ \\
& $\begin{array}{l}\text { The degree of fitness difference IS Zero AND } \\
\text { the degree of population diversity IS very small } \\
\text { The degree of fitness difference } \mathbf{I S} \text { positive small } \\
\text { AND } \\
\text { the degree of population diversity IS very small }\end{array}$ & $\begin{array}{l}\text { Mutation change rate } \\
\text { is positive medium }\end{array}$ \\
&
\end{tabular}

The composition results are shown in Table 7, Fig. 8 and Fig. 9. Using the most commonly used defuzification method, center of area method, the crisp values of the output variables are calculated. $\Delta p_{c}(t)$ gets a value of -0.04 and $\Delta p_{\mathrm{m}}(t)$ gets a value of 0.004 . Hence the new crossover rate value is 0.76 and the new mutation rate is 0.009 as calculated in formulation (21) and (22).

$$
\begin{array}{r}
p_{c}(t)=p_{c}(t-1)+\Delta p_{c}(t)=0.80-0.04=0.76 \\
p_{m}(t)=p_{m}(t-1)+\Delta p_{m}(t)=0.005+0.004=0.009
\end{array}
$$

Table 7. The composition results for the IF part of rules.

\begin{tabular}{ll}
\hline Rule No & Composition result \\
Rule 1 & $0.9966^{\wedge} 1=0.9966$ \\
Rule 2 & $0.0034^{\wedge} 1=0.0034$ \\
Rule 3 & $0.9966^{\wedge} 1=0.9966$ \\
Rule 4 & $0.0034^{\wedge} 1=0.0034$
\end{tabular}




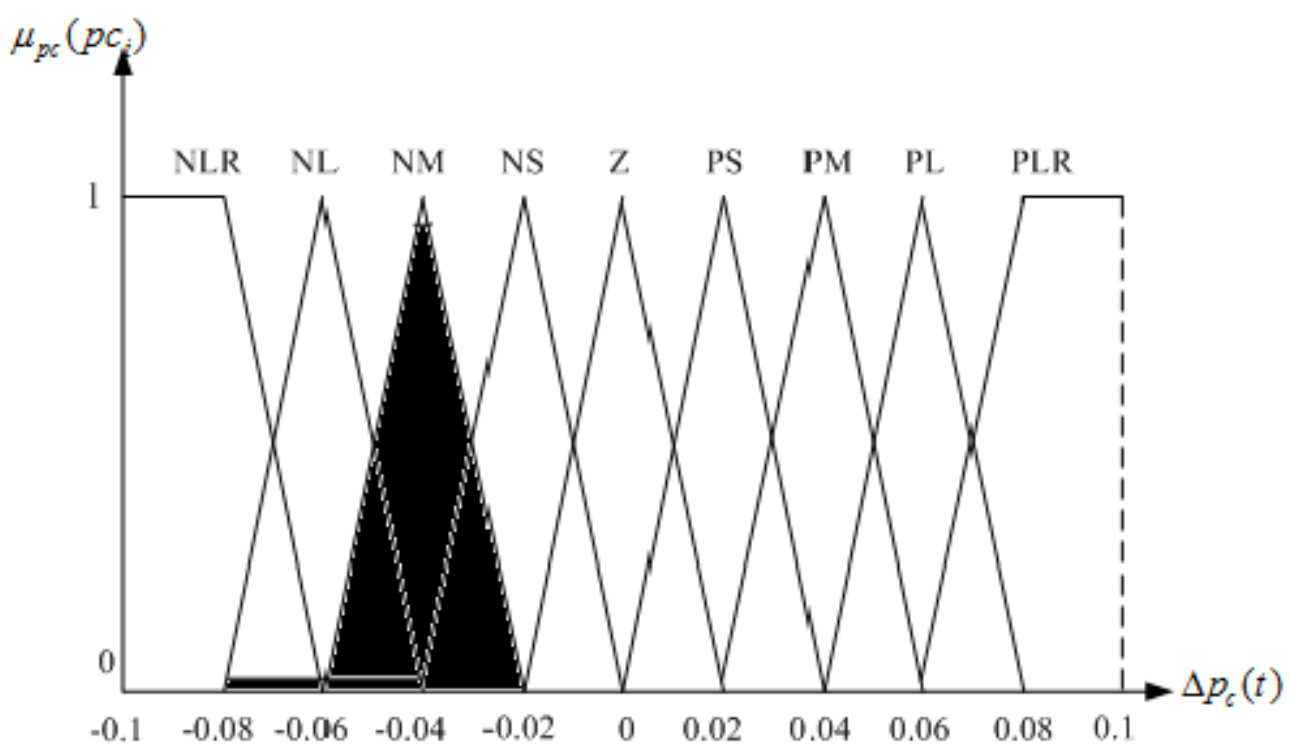

Fig. 8. Implication results of Rule $1 \&$ Rule 2

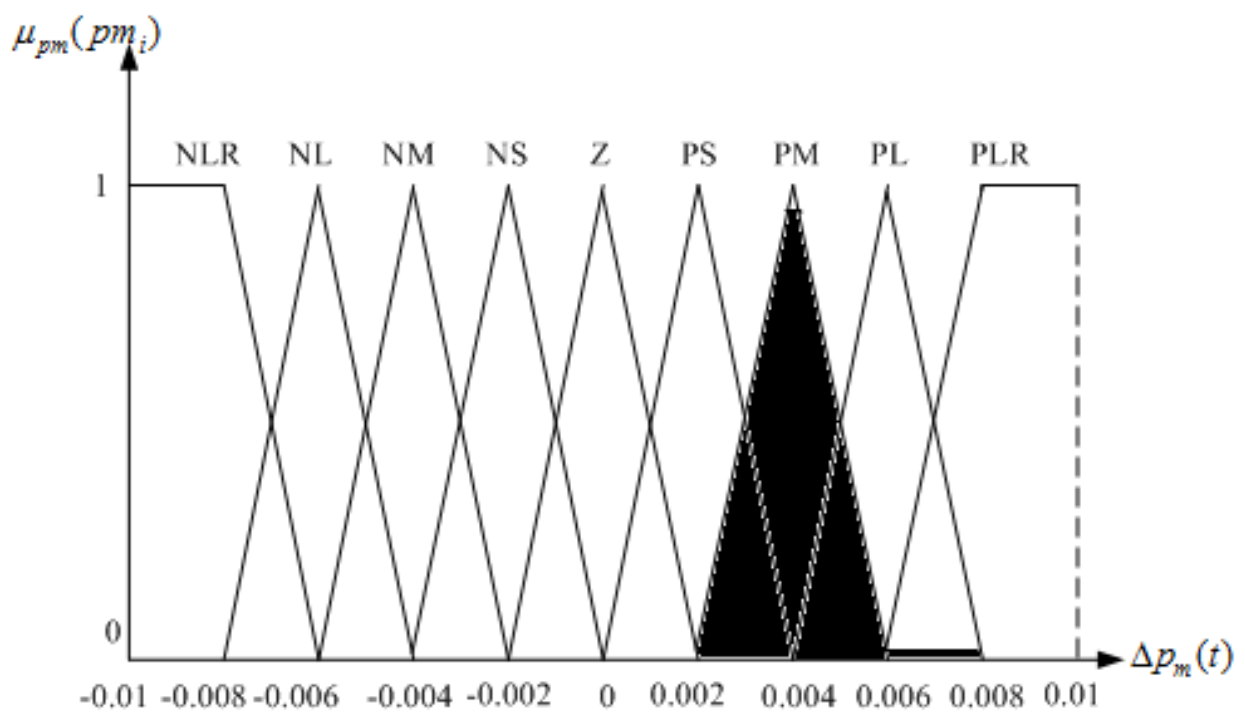

Fig. 9.Implication results of Rule $3 \&$ Rule 4

Using the new crossover rate and mutation rate, 10 simulation runs were conducted using the fuzzy based GA method and the results are shown in Table 8. The average fitness value of the 10 simulation runs is 70229, which is better than the standard GA result with a value of 72150 . The waiting passenger in each minute is reduced to around 117, showing a great increase in service level with no increase of the scheduled train. Hence, the application of the fuzzy logic approach in GA method could generate improved results. 
Table 8. Results for 10 simulation runs of fuzzy GA method.

\begin{tabular}{cccc}
\hline Runs & Fitness values & Average waiting passenger & Total number of trains \\
\hline 1 & 70347 & 128 & 11 \\
2 & 69647 & 122 & 11 \\
3 & 70213 & 102 & 12 \\
4 & 70327 & 102 & 12 \\
5 & 70987 & 132 & 11 \\
6 & 69647 & 122 & 11 \\
7 & 70913 & 107 & 12 \\
8 & 70213 & 102 & 12 \\
9 & 69647 & 122 & 11 \\
10 & 70347 & 128 & 11 \\
Average & 70228.8 & 116.7 & 11.4 \\
\hline
\end{tabular}

\section{COMPARATIVE RESULTS AND DISCUSSION}

Results in section 4 indicate that the hybrid fuzzy GA method runs better results than standard GA method. In order to further evaluate the improvement of the hybrid fuzzy GA method, Simulated Annealing (SA) approach is utilized to compare with the standard GA method and the hybrid fuzzy GA method (Zomaya, 2001). The parameters of SA are given in Table 9.

Table 9. Parameters of SA.

\begin{tabular}{ll}
\hline Parameter & Value \\
\hline Initial Temperature & 100 \\
Final temperature & 0.01 \\
Cooling factor & 0.95 \\
Length of Markov Chains & 20 \\
\hline
\end{tabular}

10 simulation runs were conducted based on different initial train schedules randomly generated in the SA model by the computer. The fitness values, average waiting passengers and number of train scheduled are shown in Table 10. 
Table 10. Results for 10 simulation runs of SA

\begin{tabular}{cccc}
\hline Runs & Fitness values & Average waiting passenger & Total number of trains \\
\hline 1 & 94060 & 230 & 14 \\
2 & 89073 & 218 & 13 \\
3 & 88833 & 266 & 11 \\
4 & 84647 & 235 & 11 \\
5 & 110367 & 453 & 10 \\
6 & 79000 & 118 & 14 \\
7 & 86447 & 223 & 12 \\
8 & 94453 & 333 & 10 \\
9 & 97027 & 328 & 11 \\
10 & 95300 & 315 & 11 \\
\hline
\end{tabular}

The average fitness value, waiting passenger and number of trains for each method are summarized in Table 11.It can be seen from Table 11 that the performance of standard GA was generally better than the performance of SA, which is partly because of the selection mechanism in GA approach. The selection mechanism of GA approach allows good-quality solutions to be chosen for mating in each generation and crossover operations produce better solutions in each generation. However, there are known limitations of the standard GA methodology, such as the premature convergence which happens because of the fixed control parameters throughout the GA run. These control parameters are chosen based on the experience of users, or some guidelines given in various studies (Lau, Chan and Tsui, 2008; Maiti 2011).

Table 11. Fitness value, waiting passenger and train schedule obtained by different approaches.

\begin{tabular}{lccc}
\hline & GA approach & $\begin{array}{c}\text { Hybrid GA fuzzy } \\
\text { approach }\end{array}$ & SA approach \\
\hline $\begin{array}{l}\text { Average fitness value } \\
\begin{array}{l}\text { Average } \\
\text { passenger }\end{array} \quad \text { waiting }\end{array}$ & 12150.8 & $\mathbf{7 0 2 2 8 . 8}$ & 91920.7 \\
Average train scheduled & 11.5 & $\mathbf{1 1 6 . 7}$ & 271.9 \\
\hline
\end{tabular}

It is also observed in Table 11 that the hybrid fuzzy GA approach has superior performances over the other approaches in fitness value, service level and number of trains, which has proved the improvement of solution quality when changing the crossover rate and mutation rate in hybrid GA fuzzy approach. The crossover rate is reduced from 0.80 to 0.76 in this scenario and the mutation ratio is increased from 0.005 to 0.009 based on the field experts' knowledge and fuzzy logic approach. The increase of the mutation rate based on the experts' tacit knowledge allows more diversity generations which in turn could lead to better results. Fuzzy logic controller enables the dynamic change of GA control parameters based on expert experience and knowledge 
of GA, which allows qualitative expression of the control strategies based on experience as well as intuition (Last and Eyal, 2005). Thus, the fuzzy GA approach is proved to have better performance than standard GA and other traditional heuristic approaches, such as SA.

\section{CONCLUSION}

There has been increasing demand for passenger rail services in recent years. How to effectively use resources to satisfy customers and in the meantime reduce cost is one of the main issues in passenger railway management. Although there are works related to passenger scheduling problem, there is limited literature investigating the adoption of practical approaches that capitalize on the merits of mathematical modeling and search algorithms for effective cost optimization. This study proposed a hybrid optimization model assisting passenger railway managers to solve the multi-objective train scheduling problem, aiming to optimize the total operational cost while maintaining a satisfactory customer service level. The hybrid fuzzy GA model proposed in this study is the seamless integration of the standard GA model and the fuzzy logic approach, both of which are well-developed computational intelligence techniques. The tacit knowledge and expertise of professional is now able to be captured in the fuzzy rules, facilitating the process of finding the optimal schedule. Based on the fuzzy rules and input values, the crossover rate and mutation rate are adjusted to enhance the performance of the search in GA. As illustrated in the numerical study, the hybrid fuzzy GA approach generates better results than both the standard GA method and SA approach in solving passenger railway scheduling problem. Thus, the adoption of the integrated fuzzy GA model is proved to be useful to passenger railway scheduling.

However, due to the complexity of passenger railway transportation, there are limitations in this study which need to be further explored. For example, this research deals with optimizing objectives such as reducing passenger waiting time and operational costs for one station only with time-varying passenger demand. Further research can be done to consider the coordination of a train service along its itinerary by considering the other stops for passenger services, and in turn, passengers' total journey times.

\section{REFERENCE}

Caprara, A.(2015). Timetabling and assignment problems in railway planning and integer multicommodity flow. Networks, 66: 1-10.

Chang, YH, Yeh, CH. \& Shen, CC. (2000). A multiobjective model for passenger train services planning: application to Taiwain's high-speed rail line. Transportation Research Part B, 34: 91-106.

Della, Croce, F., Tadei, R. \& Volta, G. (1995). A Genetic Algorithm for the Job Shop Problem. Computers \& Operations Research, 22: 15-24.

Fay, A. (2000). A fuzzy knowledge-based system for railway traffic control. Engineering application of artificial intelligence, 13: 719-729.

He, S, Song, R. \& Chaudhry, SS. (2000). Fuzzy dispatching model and genetic algorithms for railyards operations. European journal of operational research, 124 (2): 307-331. 
Huang, ZP. \& Niu, H. (2012). Study on the train operation optimization of passenger dedicated lines based on satisfaction. Discrete dynamics in nature and society, 2012: 111 .

Huisman, D. \& Kroon, LG. (2005). Operations research in passenger railway transportation. Statistica Neerlandica, 49(4):467-497.

Last, M., Eyal, S. (2005). A fuzzy-based lifetime extension of genetic algorithms. Fuzzy sets and systems, 149, 131-147.

Lau, H. C. W., Chan, T. M., Tsui, W. T., Ho, G. T. S. \& Choy, K. L. (2009). An Ai Approach for Optimizing Multi-Pallet Loading Operations. Expert Systems with Applications, 36, 4296-4312.

Lau, H.C.W. \& Dwight, R.A. (2011). A fuzzy-based decision support model for engineering asset condition monitoring - A case study of examination of water pipelines. Expert Systems with Application, 38 (10), 13342-13350.

Maiti, MK (2011). A fuzzy genetic algorithm with varying population size to solve an inventory model with credit-linked promotional demand in an imprecise planning horizon. European journal of operational research, 213: 96-106.

Niu, HM. (2011).Determination of the skip-station scheduling for a congested transit line by bilevel genetic algorithm. International journal of computational intelligence systems, 6(4): 1158-1167.

Peng, ZH, Song, B. (2010). Research on fault diagnosis method for transformer based on fuzzy genetic algorithm and artificial neural network. Kybernetes, 39(8): 1235-1244.

Schindl, D., Zufferey, N. (2015). A learning tabu search for a truck allocation problem with linear and nonlinear cost components. Naval Research Logistics , 62: 32-45.

Taleizadeh, AA, Niaki, STA, Aryanezhad, MB. \& Shafii, N. (2013). A hybrid method of fuzzy simulation and genetic algorithm to optimize constrained inventory control systems with stochastic replenishments and fuzzy demand. Information sciences, 220: 425-441.

Vromans, MJCM. \& Kroon, LG. (2004). Stochastic optimization of railway timetables, in: Proceedings TRAIL 8th Annual Congress, Delft University Press, Delft, 423- 445.

Zomaya, A.Y. (2001). Natural and simulated annealing. Computing in Science \& Engineering, 3, 97-99. 